\title{
Bioengineered Tooth-A New Horizon
}

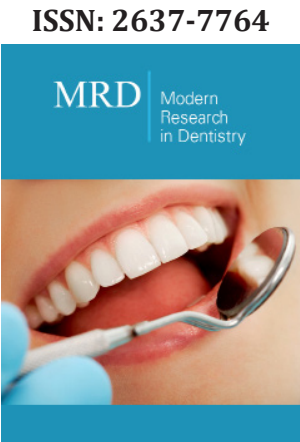

*Corresponding author: Swarga Jyoti Das, Professor \& Head, Department of Periodontics and Implantology, Regional Dental College, Guwahati-781032, Assam, India

\section{Submission: 梅 July 30, 2019}

Published: 眥 September 11, 2019

Volume 4 - Issue 3

How to cite this article: Swarga Jyot Das. Bioengineered Tooth-A New Horizon. Modern Research in Dentistry. 4(3). MRD.000589.2019.

DOI: 10.31031/MRD.2019.04.000589

Copyright@ Swarga Jyoti Das, This article is distributed under the terms of the Creative Commons Attribution 4.0 International License, which permits unrestricted use and redistribution provided that the original author and source are credited.

\author{
Swarga Jyoti Das* \\ Department of Periodontics and Implantology, India
}

\begin{abstract}
Dental mesenchymal stem cells are an attractive source of autologous stem cells. They offer a biological solution for restoring damaged dental tissues such as vital pulp and periodontal ligament and even, generation of complete or partial tooth structures to form biological implants through tissue engineering. Thus, dental tissue engineering is a new promising therapeutic approach to replace a missing tooth or to restore the damaged dental tissues. The objective of this review article is to highlight the history of the isolation of dental-related stem cells and their various types along with their potentiality and current status of applications in the field of dentistry.
\end{abstract}

\section{Introduction}

Tooth loss is a significant health issue currently affecting millions of people worldwide. It not only affects mastication and pronunciation but also leads to a series of physiological and psychological problems. The current gold standard therapy for tooth replacement is dental implants. However, it does not exhibit many properties of natural teeth and can be associated with certain complications, particularly periimplantitis, leading to implant failure [1]. Recent advancement in stem cell-based regenerative bioengineering therapy makes it possible to attain bioengineered tooth buds in experimental animals, which will be definitely a superior, promising, clinically relevant tooth replacement therapy [2,3]. It is well documented that innervation of teeth is essential for their function and protection. This can be also attained to the bioengineered teeth by autologous mesenchymal cells derived from the bone marrow with low or no immunogenicity expressed by Strub et al. [4]. Bioengineered tooth is one of the regenerative products where tissue engineering is carried out using stem cells. Stem cells are defined as clonogenic cells capable of both self-renewal and multi-lineage differentiation. They are also known as "progenitor or precursor" cells. A stem cell is essentially the building block of the human body. In 1868, the term "stem cell" for the first time appeared in the works of German biologist Haeckel. Wilson coined the term "stem cell" in [5]. These cells can be classified on the basis of their differentiation commitments (pleuripotent, multipotent and unipotent) and of their origin as embryonic, prenatal and postnatal stem cells. Postnatal stem cells, also known as the adult stem cells (ASCs), act as supportive cells by their regeneration capacity. They are primarily found in niches, namely bone marrow, skin, adipose tissue and teeth. More recently, another source of stem cells has been successfully generated from human somatic cells into a pluripotent stage. These induced pluripotent stem cells create patient- and disease-specific stem cells [6]. Among the adult stem cells, teeth are the most natural, noninvasive rich source of stem cells with decreased immunogenicity. They are easy, convenient, and affordable to collect, which are making them an attractive source of autologous stem cells. They are potential to differentiate into several cell types, including odontoblasts, neural progenitors, osteoblasts, chondrocytes and adipocytes. Considering their pluripotent natures, they are becoming a potential, novel, important tool of tissue engineering to develop biological substances. Thus, they offer a biological solution for restoring damaged dental tissues such as vital pulp, regeneration of periodontal tissue lost in periodontal disease, and for generation of complete or partial tooth structures to form biological implants [5].

\section{Dental-related stem cell biology}

There are several types of stem/progenitor cells existed in dental tissue. Depending on the sites of harvest, they can be grouped as [5,6] (Figure 1):

A. Dental Pulp Stem Cells (DPSCs)

B. Stem cells from Human Exfoliated Deciduous teeth (SHEDs) 
C. Periodontal Ligament Stem Cells (PDLSCs)

D. Dental Follicle Precursor Cells (DFPCs)

E. Stem Cells from the Apical Papilla (SCAPs)

F. Stem cells from Gingiva (GSCs)

Dental pulp stem cells (DPSCs): The presence of stem cells in dental pulp tissue primarily have been reported in 1985 by Yamamura. Later on, Caplan et al. have demonstrated that these cells presented osteogenic and chondrogenic potential invitro and could also differentiate into dentin invivo [7]. In 2000, Gronthos et al. [8] have isolated dental pulp stem cells from adult human dental pulp (Figure 2). They are capable to differentiate into odontoblasts, osteoblasts, muscle, adipose, neurons and chondrocytes invitro and can differentiate into dentin, invivo and also differentiate into dentin-pulp-like complex [8]. They show higher proliferation rate in vitro compared to that of the stem sells of other sites, which may be due to the fact that, third molars, the source, are the last permanent teeth regarding growth, formation and eruption and thus exhibits more primitive state compared to the mesenchymal stem cells of the bone marrow. Use of DPSCs in dental tissue engineering, the major issue is the scaffold. Researchers are still trying to find a competent scaffold to place DPSCs as well as the ideal microenvironment that will promote their differentiation.

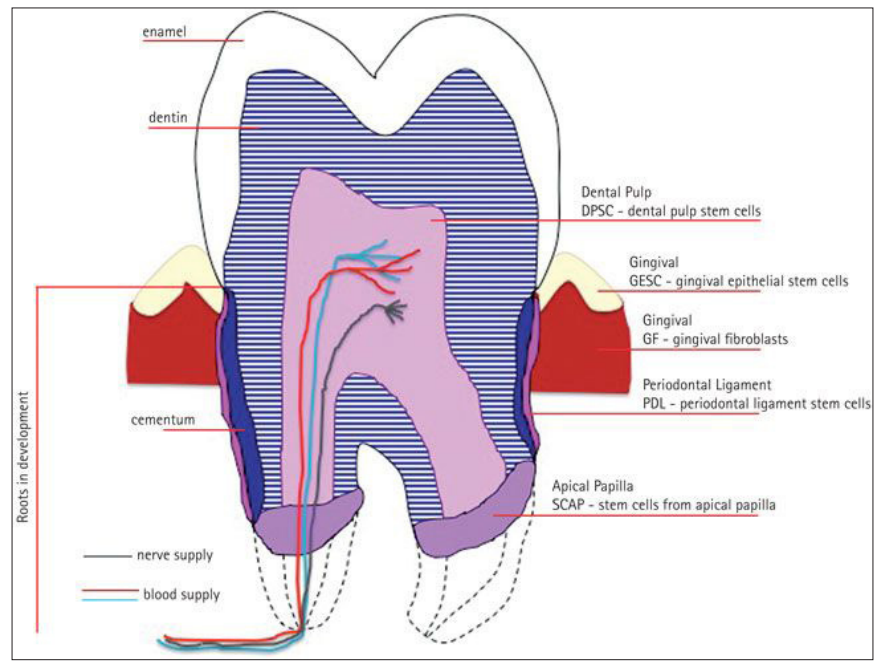

Figure 1: Sources of dental stem cells.

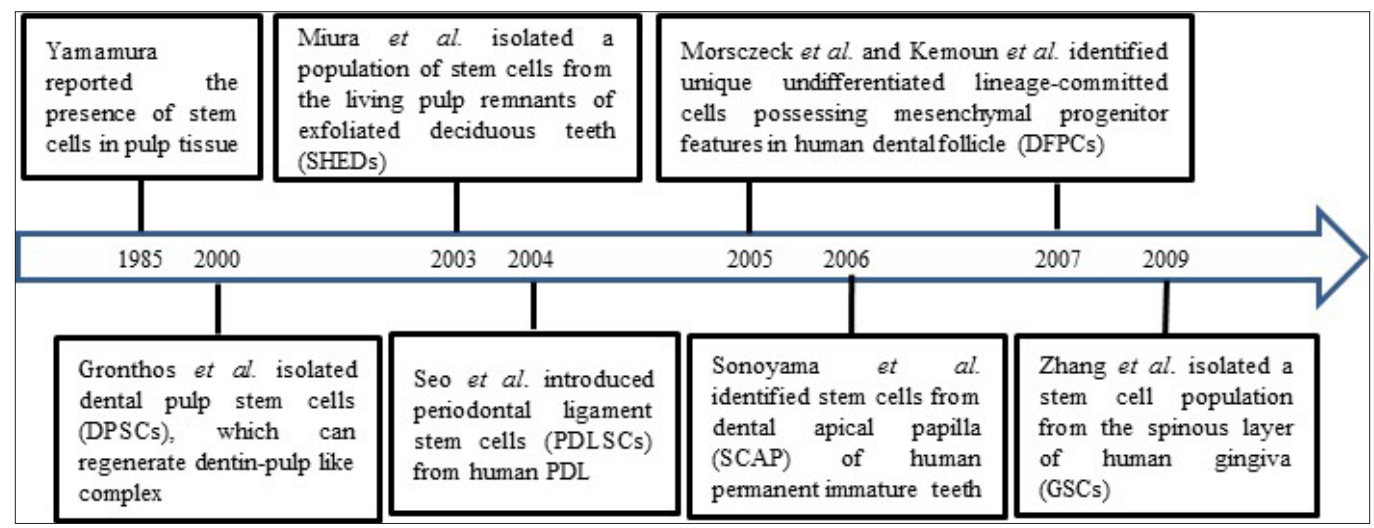

Figure 2: Timeline that highlights the history of the isolation of dental-related stem cells.

Stem cells from Human Exfoliated Deciduous teeth (SHEDs): In 2003, Miura et al. [9] have reported to isolate a stem cell population from the living pulp remnants of exfoliated deciduous teeth and they have termed the cells as stem cells from human exfoliated deciduous teeth (SHEDs) [9] (Figure 2). They have the ability to differentiate to neural cells, angiogenic endothelial cells, odontoblasts, osteoblasts, and adipocytes invitro, while have been reported to form bone and dentin like-tissue on invivo transplantation. The Developmental processes, tissue structures and functions of the deciduous teeth are different from that of permanent teeth. Thus, it is plausible that SHEDs have a higher proliferation rate, better osteo inductive ability in vivo though enable to form a dental-pulp complex.

Periodontal Ligament Stem Cells (PDLSCs): Seo et al. [10] described the presence of multipotent postnatal stem cells in the human periodontal ligament (PDL) having capacity to generate alveolar bone, cementum/periodontal ligamentlike structure [10] (Figure 2). Upon transplantation of PDLSCs into immunocompromised rodents, they generate cementum/ 
PDL-like structure and contributed to periodontal tissue repair. Formation of root and periodontal complex was observed following transplantation of hydroxyapatite/tricalcium phosphate and gel foam scaffolds seeded with autologous PDLSCs and stem cells from the dental apical papilla (SCAPs) in the alveolar bone of young pigs [11]. This newly formed root may support an artificial ceramic crown and thus, may restore the normal function of the tooth. Based on the regenerative potential of PDLSCs when seeded onto three-dimensional biocompatible scaffold, their use in graft biomaterials for bone tissue engineering in regenerative dentistry is highly recommended [12].

Dental Follicle Prescursor Cells (DFPCs): The dental follicle is a loose ectomesenchyme-derived connective tissue sac surrounding the enamel organ and the dental papilla of the developing tooth germ before eruption [13]. An unique undifferentiated lineage committed cells possessing mesenchymal progenitor features in the human dental follicle was identified by Morsczeck et al. and Kémoun et al. in 2005 and 2007, respectively (Figure 2). It is believed to contain progenitors for cement oblasts, PDL and osteoblasts [7]. These cells could be a new tool for the study of development of the periodontal ligament and for the development of regenerative therapies and reconstructive treatments.

Stem Cells from Apical Papilla (SCAPs): Mesenchymal stem cells residing in the apical papilla of permanent teeth with immature roots are regarded as SCAP. These cells were discovered by Sonoyama et al. [14] (Figure 2). They are capable of forming odontoblast-like cells, producing dentin in vivo, and are likely to be source of primary odontoblasts for the formation of root dentin, thereby, they support the apexogenesis. Important sources are of third molars and teeth with open apices. It has been demonstrated that human SCAPs possess a higher and more efficient differentiation rate compared to that of DPSCs.

Stem Cells derived from Gingiva (GSCs): The isolation of a stem cell population from gingiva was firstly reported by Zhang et al. [15]. The cells derived from the spinous layer of human gingiva are referred to as gingival stem cells (GSCs) [15] (Figure 2). Like mesenchymal stem cells, stem cells derived from Gingiva possess a differentiation potential into osteoblasts, adipocytes and chondrocytes in vitro and bone regeneration potential in vivo. Moreover, these cells have been found to be able to differentiate along endothelial as well as neural cell lineages. As with other dental related stem cells, GSCs has been found to display immunomodulatory functions, particularly the immunosuppressive factors [7].

\section{Whole tooth regeneration: interaction of epithelial and mesenchymal stem cells}

Two main cell types are involved in dental tissue formation: the ameloblasts of epithelial origin and the odontoblasts of mesenchymal origin. Enamel is formed from the ameloblasts. These cells are lost just after the eruption of a tooth, as a result they do not exist in permanent teeth and cannot, therefore, be stimulated in vivo to produce enamel [13]. Thus, dental epithelial stem cells stand as a major impediment in creation of a bio tooth. Here, induced pluripotent stem cells can be considered a cell source [16]. Dental epithelial stem cells could be isolated from the tooth germ of children's third molar and be used or saved for future use. Since this particular practice refers the child to surgery it is not ethical and may also cause immunoreactions and rejection. More research is needed for better understanding of the odontogenesis and resolve the existing impediments. However, the solution of the problem of enamel regeneration could be the use of an artificial crown, which will be supported for a teeth originating for the mesenchymal stem cells.

Several trials have been attempted for the reconstruction of teeth in vitro and in vivo using the above combination of stem cells. The bioengineered tooth germ lead to the formation of a structurally correct tooth with odontoblast's, ameloblasts, dental pulp, blood vessels, crown, periodontal ligament, root and alveolar bone. It was concluded that the implantation into the experimental animals allowed the development, the maturation and the emergence of the tooth $[2,3]$. These results are an important indication that the dental stem cells could be used for the replacement of a missing tooth in human being in near future. Successful functional tooth replacement in mouse by the transplantation of a bioengineered tooth in the area of the missing tooth in mandible is observed in 2009 by Etsuko et al. [2] with correct construction and the hardness of the tissues necessary for chewing and also respond to experimental orthodontic treatment and to noxious stimuli, such as mechanical stress and pain challenge with various thermal and electrical stimuli though the size was smaller than normal since the configuration of parameters forming the tooth, such as setting the width of the crown, is not possible with the current techniques [2]. However, the clinical application is challenging, because a tooth germ from children is required, obtaining of which is not ethical.

\section{Tooth stem cell banking}

Dental stem cell banking refers the process of storing stem cells acquired from patients' deciduous teeth and wisdom teeth. Those cells can be cryopreserved for many years to retain their regenerative capacity. Basically, it is a realization of the potential of dental stem cell-based regenerative therapy. Although tooth banking is currently not very popular, the trend is gaining acceptance mainly in the developed countries and following are few tooth stem cell banks $[5,17,18]$.

A. BioEden (Austin, Texas, USA), has international laboratories in UK (serving Europe) and Thailand (serving South East Asia) with global expansion plans.

B. Stem cell banking companies like Store-A-Tooth (Provia Laboratories, Littleton, Massachusetts, USA) and StemSave (Stemsave Inc, New York, USA) are expanding their horizon internationally.

C. In Japan, "Three Brackets" (Suri Buraketto) and one more tooth bank in Hiroshima University and Nagoya University (Kyodo, Japan) in 2005 and 2007, respectively. 
D. Taipei Medical University in collaboration with Hiroshima University opened the nation's first tooth bank in September, 2008.

E. The Norwegian Tooth Bank was set up in 2008 in Norway.

F. Stemade introduced the concept of dental stem cells banking at Mumbai and Delhi, India.

\section{Discussion}

Dental tissue engineering is a new promising therapeutic approach that aims to replace the missing tooth with a bioengineered tooth or to restore the damaged dental tissue. The ability to obtain and manipulate postnatal tissues easily from individuals to generate biologic replacement tooth materials, such as dentin, enamel, and periodontal ligament, or, even better, replace teeth of predetermined size and shape entirely, is extremely valuable [4]. Mesenchymal stem cells can be obtained with ease from dental/ oral tissue, making them an attractive source of autologous stem cells. They offer a biological solution for restoring damaged dental tissues such as vital pulp engineering, regeneration of periodontal ligament lost in periodontal disease, and for generation of complete or partial tooth structures to form biological implants. Dental mesenchymal stem cells share properties with mesenchymal stem cells from bone marrow and there is a considerable potential for these cells to be used in different fields of regenerative medicine, such as bone and muscle regeneration. In addition, their immunosuppressive properties make these cells a suitable source for treating immune disorders like systematic lupus erythematosus $[5,6,7]$. However, more research is necessary since the application of these techniques could be time-consuming, difficult and expensive and thus, may not be easily accessible to the general public.

\section{Conclusion}

A. Teeth-like structures without blood and nerve supply cannot replace actual teeth anatomically as well as functionally. Although not currently available, stem cell researches and technologies may aimed at biological alternatives to the currently used synthetic materials. The existing evidences strongly suggest the usefulness of adult stem cells aiming at repair and regeneration of various deformities, including "Bioengineered tooth buds", which will be definitely a superior alternative tooth replacement therapy than those are existing presently, and it will be a new horizon worldwide in near future.

\section{References}

1. Newman MG, Takei HH, Klokkevold PR (2011) Carranza's clinical period odontology. PA Saunders, Philadelphia, USA.
2. Ikeda E1, Morita R, Nakao K, Ishida K, Nakamura T, et al. (2009) Fully functional bioengineered tooth replacement as an organ replacement therapy. Proc Nat Acad Sci U S A 106(32): 13475-13480.

3. Smith EE, Angstadt S, Monteiro N, Zhang W, Khademhosseini A, et al. (2018) Bioengineered tooth buds exhibit features of natural tooth buds. J Dent Res 97(10): 1144-1151.

4. Strub M, Keller L, Idoux Gillet Y, Lesot H, Clauss F, et al. (2018) Bone marrow stromal cells promote innervation of bioengineered teeth. J Dent Res 97(10): 1152-1159.

5. Ramta B, Aditya J (2015) Current overview on dental stem cells applications in regenerative dentistry. J Nat Sc Biol Med 6(1): 29-34.

6. Estrela C, Alencar AH, Kitten GT, Vencio EF, Gava E (2011) Mesenchymal stem cells in the dental tissues: perspectives for tissue regeneration. Braz Dent J 22(2): 91-98.

7. Andrades JA (2013) Regenerative Medicine and Tissue Engineering. International Association of STM Publishers, Oxford, UK, pp. 95-116.

8. Gronthos S, Mankani M, Brahim J, Robey PG, Shi S (2000) Postnatal human dental pulp stem cells (DPSCs) invitro and invivo. Proc Natl Acad Sci 97(25): 13625-13630.

9. Miura M, Gronthos S, Zhao M, Lu B, Fisher LW, et al. (2003) SHED: Stem cells from human exfoliated deciduous teeth. Proc Natl Acad Sci 100 (10): 5807-5812.

10. Seo BM, Miura M, Gronthos S, Bartold PM, Batouli S, et al. (2004) Investigation of multipotent postnatal stem cells from human periodontal ligament. Lancet 364 (9429): 149-155.

11. Huang GT, Sonoyama W, Liu Y, Liu H, Wang S, et al. (2008) The hidden treasure in apical papilla: the potential role in pulp/dentin regeneration and bioroot engineering. J Endod 34(6): 645-651.

12. Trubiani O, Orsini G, Zini N, Di Iorio D, Piccirilli M, et al. (2008) Regenerative potential of human periodontal ligament derived stem cells on three-dimensional biomaterials: a morphological report. J Biomed Mater Res A 87(4): 986-993.

13. Ten cate AR (1998) oral histology-development, structure, and function. ( $5^{\text {th }}$ edn), Mosby Elsevier, St Louis, Missouri, USA, pp. 1-4.

14. Sonoyama W, Liu Y, Fang D, Yamaza T, Seo BM, et al. (2006) Mesenchymal stem cell-mediated functional tooth regeneration in swine. PLoS One 1: e79.

15. Zhang Q, Shi S, Liu Y, Uyanne J, Shi Y, et al. (2009) Mesenchymal stem cells derived from human gingiva are capable of immunomodulatory functions and ameliorate inflammation-related tissue destruction in experimental colitis. J Immunol 183(12): 7787-7798.

16. Arakaki M, Ishikawa M, Nakamura T, Iwamoto T, Yamada A, et al. (2012) Role of epithelial-stem cell interactions during dental cell differentiation. J Biol Chem 287(13): 10590-10601

17. Kaku M, Kamada H, Kawata T, Koseki H, Abedini S, et al. (2010) Cryopreservation of periodontal ligament cells with magnetic field for tooth banking. Cryobiology 61(1): 73-78.

18. Tirino V, Paino F, Aquino DR, Desiderio V, DeRosa A, et al. (2011) Methods for the identification, characterization and banking of human DPSCs: current strategies and perspectives. Stem Cell Rev Rep 7(3): 608 - 615. 